\title{
OBJECT-BASED ANALYSIS OF AERIAL PHOTOGRAMMETRIC POINT CLOUD AND SPECTRAL DATA FOR LAND COVER MAPPING
}

\author{
M. Debella-Gilo ${ }^{\text {a, }}$, K. Bjørkelo ${ }^{\text {a }}$ J. Breidenbach ${ }^{\mathrm{a}}, \mathrm{J}_{\text {. Rahlf }}^{\mathrm{a}}$ \\ ${ }^{a}$ Norwegian Forest and Landscape Institute, P.O.BOX 115, 1431 Ås, Norway - (mgd*, kbj, job, jor) \\ @skogoglandskap.no
}

KEY WORDS: Photogrammetry, Point cloud, Object-Based Image Analysis (OBIA), Land cover mapping, Image matching, Forest

\begin{abstract}
:
The acquisition of 3D point data with the use of both aerial laser scanning (ALS) and matching of aerial stereo images coupled with advances in image processing algorithms in the past years provide opportunities to map land cover types with better precision than before. The present study applies Object-Based Image Analysis (OBIA) to 3D point cloud data obtained from matching of stereo aerial images together with spectral data to map land cover types of the Nord-Trøndelag county of Norway. The multi-resolution segmentation algorithm of the Definiens eCognition ${ }^{\mathrm{TM}}$ software is used to segment the scenes into homogenous objects. The objects are then classified into different land cover types using rules created based on the definitions given for each land cover type by the Norwegian Forest and Landscape Institute. The quality of the land cover map was evaluated using data collected in the field as part of the Norwegian National Forest Inventory. The results show that the classification has an overall accuracy of about $80 \%$ and a kappa index of about 0.65 . OBIA is found to be a suitable method for utilizing 3D remote sensing data for land cover mapping in an effort to replace manual delineation methods.
\end{abstract}

\section{INTRODUCTION}

The Norwegian Forest and Landscape Institute (NFLI) produces areal resource maps with different qualities (scale and thematic details). One of such maps is the AR5 which is a land cover/use map at the scale of 1:5000. This map contains four levels of thematic information, namely: (1) the land use/cover type, (2) the dominant tree species types in forested areas (i.e. forest, grasslands and wetlands), (3) the site index which is the description of the site's productivity with respect to forest products, mainly timber, and (4) the ground condition which describes the geological and soil condition of the site.

Traditionally these maps are produced through intensive field works with the help of analogue aerial photographs until recently when manual digitization of digital orthophotos took over. It took decades to complete the economically productive part of the country (i.e. agricultural and forest areas) with the map of that scale. The Norwegian government requires that such information be updated and areas outside the economical areas need to be included. The conventional technique has become more and more costly to meet such demands. Fortunately technological developments in the areas of remote sensing and GIS in the past years and decades have created the tools and means of acquiring this information effectively creating the opportunity to take land cover mapping to a new stage.

On one hand, innovations in sensor technologies and positioning systems have led to the development of aerial laser scanning (ALS) which is nowadays widely used for acquiring 3D data with high accuracy and spatial density. ALS 3D point cloud data combined with spectral information from aerial cameras and other remote sensors have been used for land cover mapping and characterization (Antonarakis et al., 2008; Yu et al., 2011). ALS adds height information which is crucial in separating some land cover types and computing additional attributes. On the other hand, innovations in imaging sensors, image matching software and computer hardware performances have created the possibilities of acquiring images at high spatial and spectral resolutions and matching stereo images with faster speed, higher spatial precision and density. The dense matching of high spatial resolution stereo images with high precision and high spatial density can now produce good quality 3D point clouds. This is claimed to have the potential of replacing the ALS data in some applications reducing some of the costs incurred by ALS data acquisition (Leberl et al., 2010). Such high resolution data with additional third dimension requires careful selection of analysis methods.

Image analysis techniques have also progressed creating among others the possibilities of analyzing images both at supra- and sub-pixel levels. Supra-pixel analysis is necessitated when the mean object size is significantly larger than the pixel size. Supra-pixel analysis is relevant in cases such as when sub-meter spatial resolution photogrammetric images are used for land cover mapping. The best known supra-pixel image analysis method is known as Object-Based Image Analysis (OBIA). OBIA involves segmentation of images into homogeneous objects which are made of spatially contiguous pixels with minimum dissimilarity at a given scale (Blaschke, 2010). The objects can then be characterized based on a given set of attributes, and classes can be assigned to the objects using appropriate algorithms. Studies have shown that the objects are better representations of natural objects and phenomena than

\footnotetext{
* Corresponding author.
} 
pixels, and OBIA is known to produce better quality maps than pixel-based classification methods at least in 2D remote sensing (Blaschke, 2010; Hay and Castilla, 2006; Whiteside et al., 2011).

In this study the opportunities provided by such technologies are explored with the aim of updating and extending its existing land cover maps. As a result of the ever improving qualities in data and analysis methods, partial or full replacement of manual delineation of land cover types is becoming a reality. A study is thus conducted to develop methods of mapping land cover types effectively by utilizing aerial photographic images acquired for routine monitoring of Norwegian land surface. The method utilizes matching of the aerial images to create $3 \mathrm{D}$ point clouds and uses this information together with the spectral data to map land cover at detail scale by integrating class definitions used in manual delineation into automated classification. Object-based image analysis (OBIA) is employed as analysis method for the datasets. The paper continues by explaining the data and methods used followed by the discussion of the results obtained finally winding up by giving some concluding remarks.

\section{METHODS}

\subsection{Study area}

This study is conducted in the Nord-Trøndelag county of Norway (Figure 1). The county is centred approximately around $64.41^{\circ} \mathrm{N}$ and $12.00^{\circ}$ E. Forest is the dominant land cover type of this county covering over $55 \%$ of the land area covered by the AR5 map excluding water. This is followed by mire which covers about $15 \%$ of the same total area.

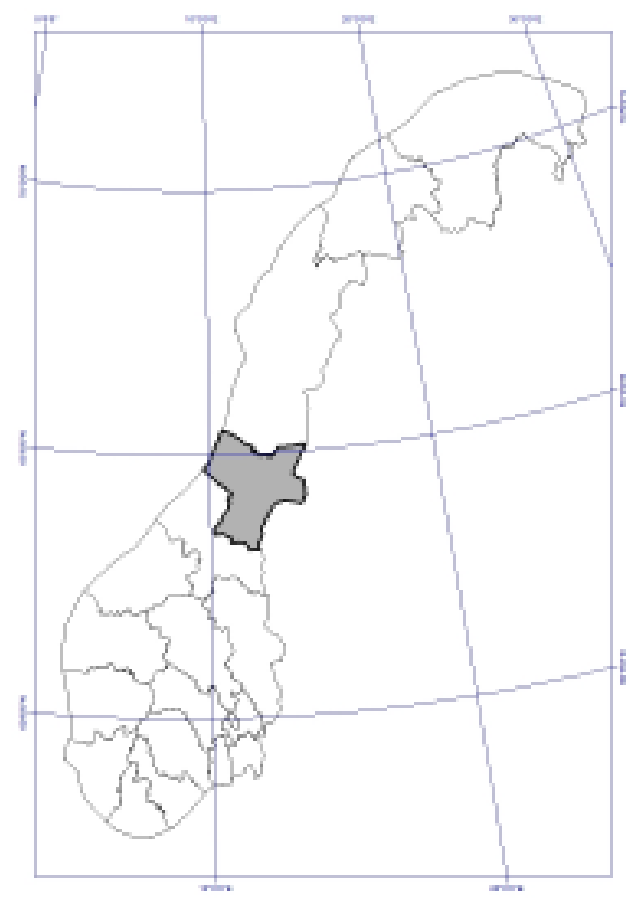

Figure 1. The location of the Nord-Trøndelag County (grey) in Norway

\subsection{Data acquisition}

Three different data sources are used in this study; namely, aerial images, digital terrain models (DTM) and field data. Each of these data sources are explained further hereunder.
2.2.1 Aerial images: The aerial images were acquired during the summer (June to September) of 2010. The ULTRACAM Xp digital camera was used for the acquisition. The images were taken with radiometric resolution of 12 bit and ground sampling distance of $35 \mathrm{~cm}$ with $60 \%$ along and $20 \%$ across track overlap. The overlapping images were matched by the BLOM GEOMATICS AS, using the software MATCH-T. The extreme terrain option with the $2.5 \mathrm{D}$ filtering was used for the matching. Hierarchical matching starting with feature-based matching and ending with block-based matching was used. The areas in which the matching failed due to occlusion or lack of contrast, the matching points were interpolated by the software. This resulted in one point per pixel, i.e. mean point density of about 9 points per $\mathrm{m}^{2}$. The point clouds together with spectral data in the red, green and infrared channels are delivered in the LAS 1.2 data format with tiles of size $1200 \mathrm{~m}$ by $1600 \mathrm{~m}$ (Figure 2).

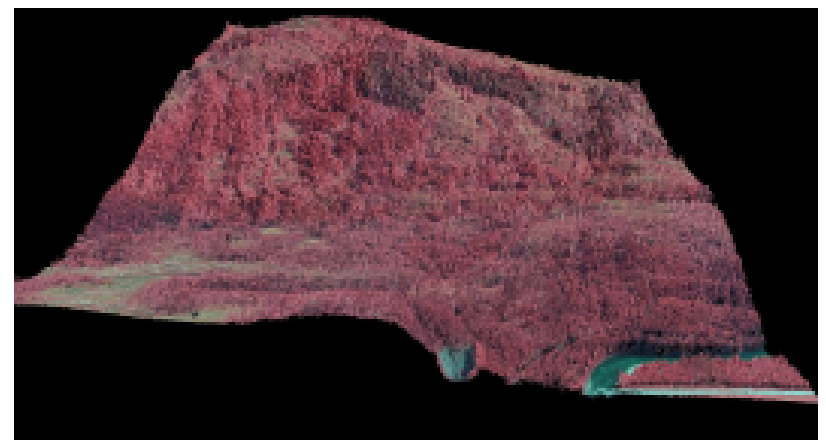

Figure 2. A 3D view of an example tile of photogrammetric point cloud with false colour (IR, R and G)

2.2.2 DTM data: the 3D point cloud data obtained from the image matching contain the digital surface model (DSM) with elevation over geodetic datum. However, it's the normalized DSM that is useful for our purpose of mapping land cover. Unlike ALS, the normalization of photogrammetric DSM necessitates the availability of accurate DTM from other sources. Two types of DTM with different qualities are available for the study area. Some part of the county is covered with a $1 \mathrm{~m}$ spatial resolution DTM derived from ALS data. The whole county is also covered with the national DTM which has a $10 \mathrm{~m}$ spatial resolution with variable and lower accuracy. Where available, the ALS DTM is used for the normalization of DTM. In other areas the national DTM is used. Slope and topographic wetness index are also computed from the national DTM data as it covers the watersheds completely.

2.2.3 Field data: automated mapping of land cover from remote sensing data necessitates understanding of the different land cover types with respect to the remote sensing data. To this end ground collect data are important. The NFLI has permanent systematic network of sample plots from which accurate ground data are collected as part of the National Forest Inventory. Each sample plot is a circle covering an area of $250 \mathrm{~m}^{2}$ with a uniform spacing of $3 \mathrm{~km}$.

\subsection{Data formats and conversion}

The photogrammetric 3D point clouds were delivered in the standard LAS data format. Each LAS file contained horizontal and vertical positions and three color bands (red, green and near infrared). The near infrared is used instead of the blue due to its greater importance in vegetation mapping. The LAS data are converted to four channel raster files in eCognition using a rule set created for this purpose. 


\subsection{Computation of CHM and NDVI}

As stated above the DSM is normalized by subtracting the digital terrain model (DTM) from the DSM. The normalized DSM is the height of all physical structures on the ground surface including vegetation, buildings, bridges, towers, etc. In forested areas, the normalized DSM is the same as the canopy height model (CHM). In areas where the ALS DTM is available, the DSM is normalized by direct subtraction of the DTM from the DSM. Outside these areas, the national DTM is interpolated to the resolution of the DSM using a bilinear interpolation algorithm and then the interpolated DSM is subtracted from the DSM. The quality of the normalized DSM or CHM is thus dependent on the type of DTM used in addition to the inherent quality of the DSM.

The normalized DSM needs to be supported by data on greenness to later identify vegetated and non-vegetated areas. The NDVI (Normalized Difference Vegetation Index), which is computed from the spectra of the red and near infrared bands, is a parameter that is widely used for this purpose. Finally both the normalized DSM and the NDVI are added to the multi-channel raster data for further analysis.

\subsection{The Object-Based Image Analysis (OBIA)}

In OBIA, the analysis units are objects rather than pixels. Objects are adjacent pixels with minimum dissimilarity in the given set of features used for the object definition and at a given scale of conceptualization. Two fundamental criteria for pixels to form an object are that they have to be adjacent to each other and their dissimilarity in the set of attributes on which object definition is based has to be below the threshold set by the scale of conceptualization. Such objects are mere aggregation of pixels. Their classes are not known until they are assigned to a certain class. Thus OBIA involves two major steps. The first is the delineation of the objects at a defined scale based on selected set of features, i.e. segmentation. The second step is the assignment of a class to each of the objects based on their feature characteristics, i.e. classification.
2.5.1 Segmentation: eCognition offers many segmentation algorithms. However, the multiresolution segmentation algorithm is chosen due to its hierarchical approach in aggregating objects at different scales. In multiresolution segmentation there are a number of parameters that need to be set. First, the features to be used have to be selected and their respective weight needs to be set. The features used in our segmentation process are the three color bands, the NDVI and the CHM. All of them are weighted equally. Second, the scale at which the objects are to be defined also needs to be set. In multi-resolution segmentation the scale parameter sets the highest permitted heterogeneity within an object, thereby determining the size of the objects. The greater the scale, the higher is the permitted heterogeneity and consequently the larger is the average object size. Third, the shape-color proportion has to be set. One of the advantages of OBIA is the fact that it can utilize the geometric properties of the objects in addition to the spectral data. We can therefore define the homogeneity criteria based on color and shape. eCognition gives options for weighting values of the shape parameter and compactness. Assuming that no ground surface resource is limited by shape, we limited the shape parameter to a mere 0.1 . That means that shape influences only $10 \%$ of the segmentation process while the spectral information and CHM control $90 \%$ of the process. It is important to recall that eCognition's scale parameter and shape-color proportion are subjective and their optimal values are obtained only through trial and error.

2.5.2 Classification: Once the scenes are segmented into homogeneous objects the next step is to assign a class to each object. Since the idea of the study is to mimic the traditional manual mapping as close as possible, the existing classification algorithms in eCognition are not used. Rather, rules that rely on the class definition of the Norwegian Forest and Landscape Institute (Bjørdal and Bjørkelo, 2006) are created. These definitions are implemented using rule sets constructed in eCognition. Class definitions in AR5 are modified to fit the remote sensing data and the hierarchical approach used here. Translating to the AR5 land cover types was done afterwards. The mire areas are identified based on the spectral data, slope and topographic wetness index. The classified objects are exported as shape files with statistics of some of the important attributes to further analyze in ARCGIS. The results are evaluated using the field data from NFI and confusion matrix is created to quantitatively present the quality of the produced land cover map. The land cover types of the NFI plots are translated from the NFI data based on the definition of each land cover type given for the AR5 map.

\section{RESULTS AND DISCUSSIONS}

As shown in Figure 3 below, good segmentation result is obtained by keeping the objects small enough to later separate different sub-types of land cover such as tree species and large enough to avoid unnecessary aggregation of different land cover types. It is observed that careful selection of the set of features used for the segmentation and the conceptualization scale is the key step in obtaining meaningful segmentation results. Meaningful segmentation here means that the boundaries of the different land cover types are segmented as precisely as possible. 

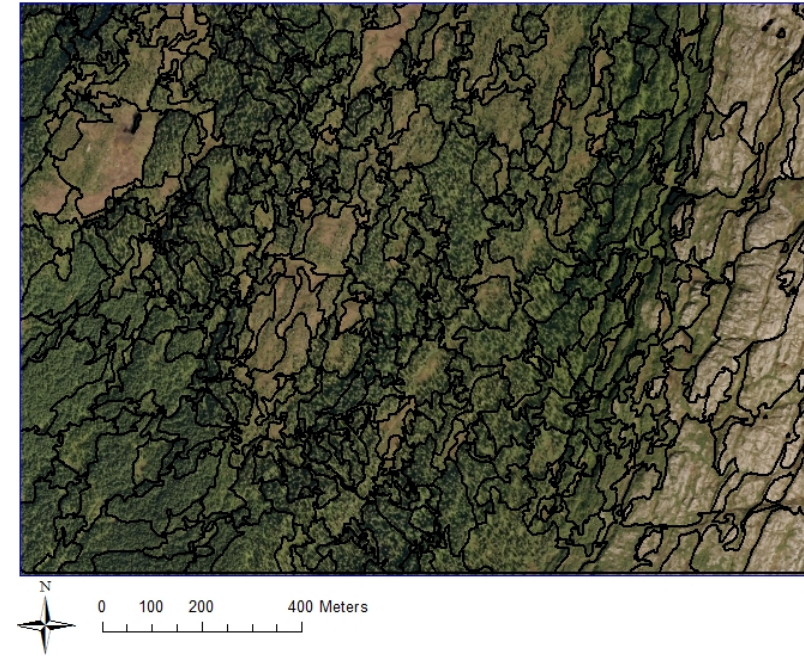

Figure 3. An example of an image tile segmented using the multiresolution algorithm

The classification resulted in four major classes outside the agricultural areas as shown, for example, in figure 4 . These are forest, mire, biotic open areas (mainly bush and grasslands) and abiotic open areas (mainly exposed rock surfaces). The open areas are split into two classes since this is required by the NFLI although they are lumped together in the existing AR5 map. The splitting of the open areas into two classes is also part of the utilization of the capabilities of remote sensing data in separating between vegetated and bare grounds. The other land cover types such as water, roads, settlements, etc. are clipped out using existing data sources.
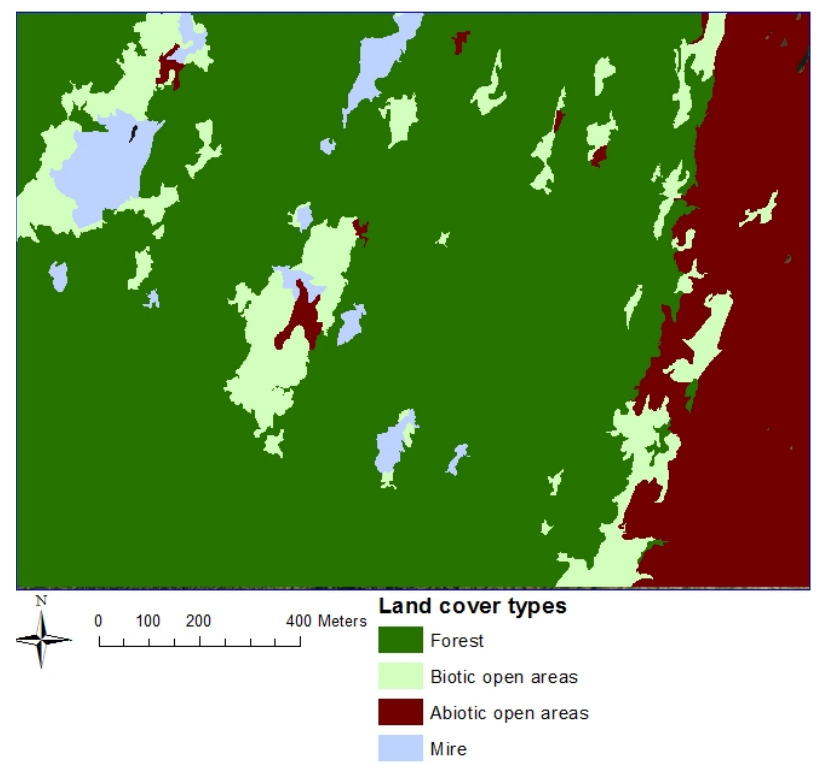

Figure 4. Classification result of the example tile

Table 1 presents the confusion matrix of the classification where the DSM is normalized using the ALS DTM. The overall accuracy and kappa index for these areas is $84 \%$ and 0.67 , respectively. Table 2 shows the confusion matrix of the areas where the DSM is normalized using the national DTM. The overall accuracy and kappa index for these areas is $72 \%$ and 0.56 , respectively. These results show that the accuracy of the classified land cover is affected by the quality of the DTM. The use of the ALS DTM with $1 \mathrm{~m}$ spatial resolution improved the overall classification accuracy by over $10 \%$. This is attributed to the fact that forest dominates the land cover type of the area and it is segmented with better precision in the first case as a result of the better quality CHM obtained when the ALS DTM is used. Note that the overall accuracy and kappa index presented in the abstract are obtained by combining the two tables.

\begin{tabular}{|c|c|c|c|c|c|c|}
\hline \multirow{2}{*}{$\begin{array}{l}\text { Actual land } \\
\text { cover type } \\
\text { translated } \\
\text { from the NFI } \\
\text { sample plots }\end{array}$} & \multicolumn{4}{|c|}{ Predicted land cover type } & \multirow{2}{*}{$\begin{array}{l}\text { Total } \\
\text { area } \\
\left(\mathrm{m}^{2}\right)\end{array}$} & \multirow{2}{*}{$\begin{array}{l}\text { Produce } \\
\mathrm{r} \\
\text { accuracy } \\
(\%)\end{array}$} \\
\hline & Forest & $\begin{array}{l}\text { Biotic } \\
\text { open } \\
\text { areas }\end{array}$ & $\begin{array}{r}\text { Abioti } \\
\mathrm{c} \\
\text { open } \\
\text { areas } \\
\end{array}$ & Mire & & \\
\hline Forest & 54012 & 2225 & 508 & 2351 & 59095 & 91 \\
\hline Open areas & 119 & 8824 & 3439 & 621 & 13003 & 94 \\
\hline Mire & 3751 & 3263 & 657 & 4339 & 12011 & 36 \\
\hline $\begin{array}{l}\begin{array}{l}\text { Total area } \\
\left(\mathrm{m}^{2}\right)\end{array} \\
\end{array}$ & 57882 & 14312 & 4603 & 7312 & & \\
\hline $\begin{array}{l}\text { User } \\
\text { accuracy (\%) }\end{array}$ & 93 & 77 & 75 & 59 & & \\
\hline
\end{tabular}

Table 1. Confusion matrix of the classification of the areas covered by the ALS DTM

Additionally, the accuracy of the classification varies from one land cover type to another. Forest has the highest accuracy whereas mire has the lowest accuracy. This can be explained by the fact that, greenness (NDVI) and canopy height model (CHM) can strongly discriminate forests. Mire however is not strongly discriminated by remote sensing and DTM data. The distribution of mire is dependent on geological drainage and the availability of sphagnum vegetation which are not easily detectable by the remote sensing data used here. Besides, the lack of accurate DTM for the computation of slope and topographic wetness index may have led to the poor prediction of the mire areas.

In general the classification accuracy is found to be satisfactory for forest and the two types of open areas implicating that the method can be used to update and extend the existing land cover maps. However, other approaches are needed to improve the quality of the prediction of the areas covered by mire. The accuracy tables should be considered with caution as the areas used for the accuracy evaluation are much smaller in relation to the total area covered by the study.

\begin{tabular}{|l|r|r|r|l|l|l|}
\hline \multirow{2}{*}{$\begin{array}{l}\text { Actual land } \\
\text { cover type } \\
\text { translated } \\
\text { form NFI } \\
\text { sample plots }\end{array}$} & \multicolumn{4}{|c|}{ Predicted land cover type } & $\begin{array}{l}\text { Total } \\
\text { area } \\
\left(\mathrm{m}^{2}\right)\end{array}$ & $\begin{array}{l}\text { Produc } \\
\text { er } \\
\text { accurac } \\
\text { y (\%) }\end{array}$ \\
\cline { 2 - 5 } & Forest & $\begin{array}{r}\text { Biotic } \\
\text { open } \\
\text { areas }\end{array}$ & $\begin{array}{r}\text { Abioti } \\
\text { c } \\
\text { open } \\
\text { areas }\end{array}$ & Mire & & \\
\hline Forest & 43601 & 7248 & 1255 & 3410 & 55513 & 80 \\
\hline Open areas & 7777 & 16688 & 17534 & 6917 & 48916 & 70 \\
\hline Mire & 2410 & 4166 & 3864 & 12932 & 23371 & 55 \\
\hline $\begin{array}{l}\text { Total area } \\
\left.\text { (m }{ }^{2}\right)\end{array}$ & 53788 & 28102 & 22652 & 23259 & & \\
\hline $\begin{array}{l}\text { User } \\
\text { accuracy (\%) }\end{array}$ & 80 & 60 & 77 & 56 & & \\
\hline
\end{tabular}

Table 2. Confusion matrix of the classification of the areas the CHM is computed using the national DTM

\section{CONCLUSIONS}

The study shows that photogrammetric point cloud data supplemented by spectral information can produce good quality land cover maps at very detailed scale. The addition of the height information particularly improved the separation of 
forests from other vegetated areas. However, a DTM with high accuracy and spatial resolution is important in improving the result of the classification. OBIA is found to be a well suited method for the segmentation and classification of land cover types using such high spatial resolution data. The segmentation of the images prior to classification removes the salt-and-pepper patterns known to be common in pixel-based classification. The fact that good quality map can be produced without using the in-built classification algorithms but rules based on the class definitions created for manual mapping shows that we are getting much closer to replacing manual delineation of land cover by automated remote sensing approach.

The project will further continue to estimate the tree species types and site quality index of the forest segments based on the spectral, canopy height, NDVI, terrain and other auxiliary data. Additional information such as mean biomass, volume and mean canopy height can also be computed for the forest areas using methods presented in (Breidenbach and Astrup, 2012; Breidenbach et al., 2010). This has implications as, for example, photogrammetric stereo images could be utilized to understand the expansion and growth rate of forests.

\section{REFERENCES}

Antonarakis, A.S., Richards, K.S., Brasington, J., 2008. Objectbased land cover classification using airborne LiDAR. Remote Sensing of Environment, 112(6): 2988-2998.

Bjørdal, I., Bjørkelo, K., 2006. AR5 Klassifikassjonssystem: Klassification av arealressurser, Norsk Institutt for Skog og Landskap, Ås, Norway.

Blaschke, T., 2010. Object based image analysis for remote sensing. Isprs Journal of Photogrammetry and Remote Sensing, 65(1): 2-16.

Breidenbach, J., Astrup, R., 2012. Small area estimation of forest attributes in the Norwegian National Forest Inventory. European Journal of Forest Research, 131(4): 1255-1267.

Breidenbach, J., Næsset, E., Lien, V., Gobakken, T., Solberg, S., 2010. Prediction of species specific forest inventory attributes using a nonparametric semiindividual tree crown approach based on fused airborne laser scanning and multispectral data. Remote Sensing of Environment, 114(4): 911-924.

Hay, G., Castilla, G., 2006. Object-based image analysis: strengths, weaknesses, opportunities and threats (SWOT), Proc. 1st Int. Conf. OBIA.

Leberl, F. et al., 2010. Point Clouds: Lidar versus 3D Vision. Published in Photogrammetric Engineering and Remote Sensing, 76(10): 1123.

Whiteside, T.G., Boggs, G.S., Maier, S.W., 2011. Comparing object-based and pixel-based classifications for mapping savannas. International Journal of Applied Earth Observation and Geoinformation, 13(6): 884893.

Yu, H.-y., Cheng, G., Ge, X.-s., Lu, X.-p., 2011. Object oriented land cover classification using ALS and GeoEye imagery over mining area. Transactions of Nonferrous Metals Society of China, 21, Supplement 3(0): s733-s737. 